\title{
Static and dynamic evaluation of pelvic floor disorders with an open low-field tilting magnet
}

\author{
V. Fiaschetti ${ }^{\text {a }}$, D. Pastorelli ${ }^{\text {a }}$, E. Squillaci ${ }^{\text {a }}$, V. Funel ${ }^{\mathrm{a}, *}$, M. Rascioni ${ }^{\mathrm{a}}$, A. Meschini $^{\mathrm{a}}$, \\ C. Salimbeni ${ }^{\text {a }}$, P. Sileri ${ }^{\text {b }}$, L. Franceschilli ${ }^{b}$, G. Simonetti ${ }^{\text {a }}$

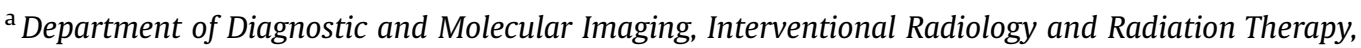 \\ University of Rome "Tor Vergata", Rome, Italy \\ b Surgery Department, University of Rome "Tor Vergata”, Rome, Italy
}

\section{ARTICLE INFORMATION}

\section{Article history:}

Received 20 May 2012

Received in revised form

23 November 2012

Accepted 26 November 2012
AIM: To assess the feasibility of magnetic resonance defaecography (MRD) in pelvic floor disorders using an open tilting magnet with a $0.25 \mathrm{~T}$ static field and to compare the results obtained from the same patient both in supine and orthostatic positions.

MATERIALS AND METHODS: From May 2010 to November 2011, 49 symptomatic female subjects (mean age 43.5 years) were enrolled. All the patients underwent MRD in the supine and orthostatic positions using three-dimensional (3D) hybrid contrast-enhanced (HYCE) sequences and dynamic gradient echo (GE) T1-weighted sequences. All the patients underwent conventional defaecography (CD) to correlate both results. Two radiologists evaluated the examinations; inter and intra-observer concordance was measured. The results obtained in the two positions were compared between them and with $\mathrm{CD}$.

RESULTS: The comparison between CD and MRD found statistically significant differences in the evaluation of anterior and posterior rectocoele during defaecation in both positions and of rectal prolapse under the pubo-coccygeal line $(\mathrm{PCL})$ during evacuation, only in the supine position (versus MRD orthostatic: rectal prolapse $p<0.0001$; anterior rectocoele $p<0.001$; posterior rectocoele $p=0.008$; versus $\mathrm{CD}$ : rectal prolapse $p<0.0001$; anterior rectocoele $p<0.001$; posterior rectocoele $p=0.01$ ). The value of intra-observer intra-class correlation coefficient (ICC) ranged from good to excellent; the interobserver ICC from moderate to excellent.

CONCLUSION: MRD is feasible with an open low-field tilting magnet, and it is more accurate in the orthostatic position than in the supine position to evaluate pelvic floor disorders.

(c) 2012 The Royal College of Radiologists. Published by Elsevier Ltd. All rights reserved.

\section{Introduction}

The pelvic floor disorders (PFD), including pelvic organ prolapse, stress urinary incontinence, over-reactive bladder, obstructive defaecation syndrome, and faecal incontinence

\footnotetext{
* Guarantor and correspondent: V. Funel, Department of Diagnostic and Molecular Imaging, Interventional Radiology and Radiation Therapy, University of Rome "Tor Vergata", Viale Oxford 81, 00133 Rome, Italy. Tel.: +39 (0) 620902400; fax: +39 (0) 620902404.

E-mail address: valefunel@hotmail.it (V. Funel).
}

are increasingly common and have a significant impact on quality of life as well as an economic burden. The common final pathway of this set of disorders is the relaxation of the supporting tissue of the pelvic viscera, and usually has multiple causes, including risk factors such as age, vaginal delivery (especially instrumented), multiparity, chronic straining, connective tissue disease, congenital defects, pelvic neuropathies, and pelvic surgery.

The prevalence of PFD is reported as high as $30 \%$ in the adult population, ranging mainly between 10 and 15\%; 
despite this, the number might be underestimated. Up to $10 \%$ of women in the USA need a surgical correction of some PFD during their life, confirming that many cases of PFD are often undiagnosed. ${ }^{1}$

The abnormality may affect one or more pelvic floor compartments, often resulting in a prolapse and/or a disorder of the bladder (urinary incontinence and other urinary disorders), of the vagina and/or the uterus (sexual disorders) and the rectum (faecal incontinence and chronic constipation).

The surgical planning is based essentially on physical examination, on urinary and faecal functional testing and on conventional defaecography (CD). The information obtained from available techniques are compared and integrated with magnetic resonance defaecography (MRD) to increase diagnostic accuracy and provide a complete case history. $^{2}$

Magnetic resonance imaging (MRI) still plays an experimental role in the study of both defaecation and PFD, but it offers the unquestionable advantages of not exposing patients to unnecessary radiation (especially women of child-bearing age), and characterizing the pelvic floor muscles, which are otherwise only indirectly visualized by standard proctograms.

Until now, superconductive magnets with a static field greater than $0.5 \mathrm{~T}$ have been used in all the existing publications comparing CD and MRD; supine or sitting dedicated positions are reported. ${ }^{3,4}$ Moreover all studies of MRD, either in the supine or sitting position, used different MRI systems with different magnetic fields. ${ }^{5}$

The present study evaluated the feasibility of MRD in patients with PFD using an open permanent magnet with a changeable position and a static $0.25 \mathrm{~T}$ field. The results obtained with MRD were compared in the same subject in the supine and in the orthostatic positions; the results of MRD were also compared with CD. The MR system used in this research allowed a direct comparison between data series of the two different positions avoiding the bias due to different technical conditions.

\section{Materials and methods}

\section{Patient population}

From May 2010 to November 2011, 49 consecutive patients who had symptoms of chronic constipation, feeling of incomplete evacuation, pain during defaecation, and/or faecal incontinence were enrolled. All the patients were referred by certified colorectal surgeons and underwent prior outpatient examinations, including digital examination and proctoscopy. All the patients were females aged between 22 and 65 years (mean age 43.5 years; Table 1 ). All the patients gave informed consent. The study was performed according to the standards of the Declaration of Helsinki; approval of the local ethical committee had been granted as part of an ongoing study. ${ }^{6}$

One patient had previously undergone stapled trans-anal rectal resection (STARR) for obstructed defaecation and
Table 1

Clinical symptoms reported by the patients examined.

\begin{tabular}{lc}
\hline Symptom & No. of patients \\
\hline Feeling of incomplete evacuation & $35 / 49$ \\
Pain during defaecation & $7 / 49$ \\
Faecal incontinence & $10 / 49$ \\
Chronic constipation & $41 / 49$ \\
Sense of rectal bulging & $18 / 49$ \\
Dyspareunia & $14 / 49$ \\
Sense of vaginal bulging & $10 / 49$ \\
Feeling of incomplete urination & $6 / 49$ \\
Dysuria & $10 / 49$ \\
Sense of vesical bulging & $3 / 49$ \\
\hline
\end{tabular}

rectocoele, and four others had undergone a hysterectomy to resect a fibroid uterus. Three patients were nulliparous, whereas the remaining women had a mean of 1.3 children.

All 49 patients gave their consent to undergo a colpocysto-defaecography or $\mathrm{CD}$. These were performed by the same radiologists (V.F. and D.P.), but on a different date from the MRD.

\section{Imaging technique}

\section{$M R D$}

MRD was performed using a permanent open magnet with changeable positions and static $0.25 \mathrm{~T}$ field, dynamic gradients with $20 \mathrm{mT} / \mathrm{m}$ power and $25 \mathrm{mT} / \mathrm{m} / \mathrm{s}$ slew rate (G-SCAN, Esaote S.p.A., Genova, Italy). The magnet table was provided with a tilting mechanism from $0^{\circ}$ to $90^{\circ}$ with $2^{\circ}$ steps, and allowed the evaluation both in supine and orthostatic positions. A surface lumbar spine DPA coil was used as the receiving coil, composed of a stiff base (length $320 \mathrm{~mm} \times$ depth $280 \mathrm{~mm} \times$ height $45 \mathrm{~mm}$ ) and a flexible anterior band with variable dimensions (big band $89 \times 18.5 \mathrm{~cm}$; little band $69 \times 18.5 \mathrm{~cm}$ ) depending on the size of each subject. The protocol used was developed in a previous pilot study performed at the authors' institution. $^{6}$

Before the examination, the rectum was filled with approximately $200 \mathrm{ml}$ of suspension media (mashed potatoes) mixed with $1 \mathrm{ml}$ paramagnetic contrast media gadobutrol (Gadovist $1 \mathrm{~mol} / \mathrm{l}$, Schering AG, Berlin, Germany).

The bladder was also filled with $180 \mathrm{ml}$ physiological solution mixed with $3 \mathrm{ml}$ paramagnetic contrast media gadobutrol (Gadovist $1 \mathrm{~mol} / \mathrm{l}$ ) via a $16 \mathrm{~F}$ double-way Foley catheter, which remained in place during the entire study. Finally, the vagina was filled with an echographic gel suspension (Aquasonic 100, Parker Laboratories, Fairfield, NJ, USA) mixed with $0.5 \mathrm{ml}$ paramagnetic contrast media gadobutrol. The mean time required to prepare the patient was $20 \mathrm{~min}$ (range $14-27 \mathrm{~min}$ ). Initially the examination was performed in the orthostatic position, with the magnet table pitched at $80^{\circ}$.

The three orthogonal image planes, were acquired at rest using the 3D HYCE sequence (hybrid contrast enhanced), a type of gradient echo balanced sequence with the following characteristics: $10 \mathrm{~ms}$ repetition time (TR); $5 \mathrm{~ms}$ echo time (TE); $90^{\circ}$ Flip Angle; 20 section; $2.5 \mathrm{~mm}$ section thickness; $280 \times 280$ field of view; $200 \times 160$ matrix. 
The static images were acquired in the sagittal plane at rest, and during sphincter contraction and straining using a GE T1-weighted sequence with the following parameters: $35 \mathrm{~ms}$ TR; $10 \mathrm{~ms} \mathrm{TE}$; $90^{\circ}$ flip angle; one section; $5.5 \mathrm{~mm}$ section thickness; $300 \times 300$ FOV; $192 \times 128$ matrix.

Finally, the dynamic phase was performed during defaecation using a GE T1-weighted sequence in the sagittal plane and with the following parameters: $30 \mathrm{~ms}$ TR; $6 \mathrm{~ms}$ TE; $90^{\circ}$ flip angle; one section; $5.5 \mathrm{~mm}$ section thickness; $300 \times 300$ FOV; $192 \times 128$ matrix; 3 s/image acquisition time. T2-weighted sequences were not available. The same study protocol was followed in the supine position with the magnet table pitched at $0^{\circ}$, prior to second filling of the rectal ampulla.

Finally, the bladder catheter was removed and the urinary study was performed using the same dynamic sequences used in the defaecation study.

The overall magnet time required to complete the acquisition in both positions and the second rectal filling was an average of $68 \mathrm{~min}$ (range 42-93 $\mathrm{min}$ ).

\section{Colpo-cysto-defaecography}

The colpo-cysto-defaecography was acquired on a remote-controlled digital radiological system OPERA T90cex (General Medical, Merate, Italy) in the sitting position through a dedicated radio-transparent device.

The pelvic organs were prepared as follows: the vagina was filled with $50 \mathrm{ml}$ echographic gel (Aquasonic 100, Parker Laboratories, Fairfield, NJ, USA) mixed with $5 \mathrm{ml}$ iodinated contrast media (iopamidol, $370 \mathrm{mg}$ iodine $/ \mathrm{ml}$; Iopamiro 370, Bracco S.p.A., Milano, Italy); the bladder was filled with $120 \mathrm{ml}$ physiological solution and $120 \mathrm{ml}$ iopamidol (Iopamiro 370) through a $16 \mathrm{~F}$ double-way bladder Foley catheter, left in situ; the rectum was filled with 180-240 ml barium paste (Prontobario 110\% p/v, Bracco S.p.A, Milano, Italy); the ileal loops were filled with $250 \mathrm{ml}$ oral barium solution (Prontobario HD, Bracco S.p.A., Milano, Italy), about $45 \mathrm{~min}$ before the examination.

At first, static images were acquired in the lateral, anteroposterior, and oblique projections, respectively, at rest, and during contraction, and straining, and then we performed a dynamic defaecatory phase acquired only in the lateral projection using serial imaging (1-3 images/s).

After removing the bladder catheter, dynamic images were acquired using serial imaging (1-3 images/s) in the oblique projection in order to evaluate the urinary function. The technical parameters were the following: $80-90 \mathrm{kV}$, $100 \mathrm{mAs}$ and focus-sensitive plain distance $1.10 \mathrm{~m}$.

\section{Imaging analysis}

Only the images acquired in the sagittal plane were used for quantitative analysis, as grading systems are based on the bidimensional sagittal images obtained with CD. All examinations were evaluated separately by two radiologists to establish inter-observer concordance. Both observers were experienced in PFD study (3 years of experience for the first observer, 1 year of experience for the second) and they repeated the measurements 1 month later to evaluate intra-observer concordance. The aim of image analysis was to determine the presence and severity of enterocoeles, anterior and posterior rectocoeles, and rectal vaginal and bladder prolapses.

According to the literature, both the pubo-coccygeal line $(\mathrm{PCL})$ and the HMO system ( $\mathrm{H}$ line, $\mathrm{M}$ line and pelvic organ prolapse below the $\mathrm{H}$ line) were used in order to determine and assess the severity of pelvic organ prolapse as well as pelvic floor weakness. $2,4,5$

All the measurements were made at rest, and during straining and contraction in the two different positions. Measurements were obtained using the following anatomical landmarks: the PCL, defining pelvic floor base, extending from the inferior border of pubic symphysis to the last visible coccygeal joint. The distance between the PCL and the lowest recognizable part of pelvic organs (bladder, urethra, posterior vaginal fornix, rectum, and small intestine), measured at rest, and during straining and defaecation provided quantitative assessment of pelvic organ prolapse according to severity degrees (Table 2 ) $^{7}$; the $\mathrm{H}$ line, index of the widening of pubo-rectal hiatus, was measured by drawing a line from the inferior border of the pubic symphysis to the posterior border of the pubo-rectalis muscle (representing the lower part of the muscular group of levator ani). According to the literature, abnormal widening of the pubo-rectal hiatus was defined as the $\mathrm{H}$ line $>6 \mathrm{~cm}$ (Table 3); the M line, an index of pelvic floor descent, was measured by drawing a line perpendicular to the $\mathrm{PCL}$ from the posterior border of the $\mathrm{H}$ line. Abnormal descent was occurred when the length of the $M$ line was greater than $2 \mathrm{~cm}$ (Table 3 ).

According to the HMO system, prolapse was considered as descent of a pelvic organ (bladder, urethra, posterior vaginal fornix, rectum, and small bowel) through the puborectal hiatus ( $\mathrm{M}$ line). Evaluation of pelvic organ prolapse severity was obtained from the shortest distance between the inferior border of the prolapsed organ and the $\mathrm{H}$ line (Table 4$)^{8}$

The ano-rectal angle (ARA) corresponds to the angle measured at the intersection between the line tangent to the posterior wall of the rectum and a line parallel to the axis of the anal canal and the ano-rectal junction (ARJ) corresponds to the vertex of this intersection. In normal conditions, the ARA lies at rest $<3 \mathrm{~cm}$ from the PCL and measures $108-127^{\circ}$. This angle is an index of the function of the pubo-rectalis muscles. During maximal contraction this angle usually becomes $15-20^{\circ}$ sharper than at rest; according to the literature, a change in this angle inferior or equal to $10^{\circ}$ was considered to be a pathological change. During straining and defaecation, instead, the pubo-rectalis muscles relax and the ARA become $15-20^{\circ}$ more obtuse,

Table 2

Classification of severity of pelvic organs prolapse below pubo-coccygeal line (PCL).

\begin{tabular}{ll}
\hline Grade & Descent below the PCL $(\mathrm{cm})$ \\
\hline 0 (normal) & $0-1$ \\
1 (mild) & $1-3$ \\
2 (moderate) & $3-6$ \\
3 (severe) & $>6$ \\
\hline
\end{tabular}


Table 3

Classification of severity of pelvic floor weakness on the basis of pubo-rectal hiatus enlargement ( $\mathrm{H}$ line) and of pelvic floor descent ( $\mathrm{M}$ line).

\begin{tabular}{lll}
\hline Grade & $\begin{array}{l}\text { H line }(\mathrm{cm}) \\
\text { (hiatal enlargement) }\end{array}$ & $\begin{array}{l}\text { M line }(\mathrm{cm}) \\
\text { (pelvic floor descent) }\end{array}$ \\
\hline 0 (normal) & $<6$ & $<2$ \\
1 (mild) & $6-8$ & $2-4$ \\
2 (moderate) & $8-10$ & $4-6$ \\
3 (severe) & $\geq 10$ & $\geq 6$ \\
\hline
\end{tabular}

with a widening that must not exceed $15-20^{\circ}$ from the basal condition. . $^{10}$

The rectocoele, either anterior or posterior, was evaluated by drawing a line parallel to the anterior or posterior wall of the anal canal and measuring the distance between this line and the widest point of bulging (Table 5). ${ }^{5}$

Using $C D$ the organ prolapse under the PCL, the width of the ARA, and possible rectocoeles were measured, establishing an analogous severity class as for the MR examination.

\section{Statistical analysis}

Due to the paucity of abnormal findings in the anterior and middle compartments diagnosed in the present patient population, statistical analysis was limited to assessment of the posterior compartment abnormalities. Analysis of variance (ANOVA) was used to compare the measurements of rectal prolapses, and anterior and posterior rectocoeles obtained from the MR images acquired in the orthostatic and supine positions and from $\mathrm{CD}$. The evaluation of the ARA was based on the results of maximal contraction. The groups were compared using the Wilcoxon sign test. The intraclass correlation coefficient (ICC), both interobserver and intraobserver, was used to establish the repeatability of measurements obtained from MRD in the orthostatic position, at rest and during straining and defaecation.

Concordance was considered excellent for the ICC values higher than 0.8; good for the ICC values between 0.6 and 0.8 , moderate between 0.4 and 0.6 and poor for the values lower than 0.4 .

\section{Results}

According to the measurements of the MRD orthostatic (MRDo) images, abnormalities of different severity classes were observed in present patient population (Table 6). Among the 49 patients examined, 15 showed rectal mucosa intussusceptions (Fig 1), 16 vaginal prolapses, one enterocoele, and one omentocoele behind the PCL were found.

Table 4

Severity classification of pelvic organs prolapse below the $\mathrm{H}$ line.

\begin{tabular}{ll}
\hline Grade & Prolapse below the $\mathrm{H}$ line $(\mathrm{cm})$ \\
\hline 0 (normal) & 0 \\
1 (mild) & $0-2$ \\
2 (moderate) & $2-4$ \\
3 (severe) & $>4$ \\
\hline
\end{tabular}

Table 5

Severity classification of rectocoele.

\begin{tabular}{ll}
\hline Grade & Rectocoele $(\mathrm{cm})$ \\
\hline 1 (mild) & $<2$ \\
2 (moderate) & $2-4$ \\
3 (severe) & $>4$ \\
\hline
\end{tabular}

Also four rectal diverticula and one uretral diverticulum were found. In two cases a rectal fistula was detected.

The comparison made between $\mathrm{CD}$ and the MR examinations acquired in each position showed statistically significant differences in the evaluation of the three main abnormalities (rectal prolapse $p<0.0001$; posterior rectocoele $p<0.0001$; anterior rectocoele $p<0.001$ ).

In particular, the values found in supine MRD (MRDs) showed an underestimation of the values compared both to the MRDo (rectal prolapse $p<0.0001$; anterior rectocoele $p<0.001$; posterior rectocoele $p=0.008$ ) and to CD (rectal prolapse $p<0.0001$; anterior rectocoele $p<0.001$; posterior rectocoele $p=0.01$; Figs $1-3$; Table 7).

In the present study, the intra-observer concordance was good to excellent for all measurements obtained. In particular, for the ARJ prolapse below the PCL the ICC values were 0.92 at rest and 0.94 both during straining and defaecation, whereas for prolapses under the $\mathrm{H}$ line and $\mathrm{M}$ line, the ICC was 0.68 and 0.76 at rest, 0.78 and 0.78 during straining, and 0.76 and 0.75 during evacuation. The ICC value for rectal descent below the $M$ line was 0.71 and 0.69 during straining and defaecation, respectively. The ICC results for the ARA was 0.87 at rest, 0.85 during straining, 0.89 during maximal contraction, and 0.87 during evacuation. The measurements of the anterior rectocoele showed an ICC of 0.87 during straining and 0.9 during defaecation.

The results of the interobserver concordance for all the measurements were from moderate to excellent with lower absolute values compared to intra-observer ICC values. In particular, the ICC for the ARJ descent below the PCL was 0.85 at rest, 0.81 during straining, and 0.84 during defaecation. The concordance regarding measurements of the $\mathrm{H}$ and $\mathrm{M}$ lines was moderate with ICC values of 0.60 and 0.44 at rest; 0.46 and 0.48 during straining; and 0.55 and 0.52 during evacuation, respectively. Similar results were observed in the evaluation of rectal prolapses below the $\mathrm{H}$ line with the ICC values during straining and defaecation of 0.40 and 0.42 , respectively.

\section{Table 6}

Results divided into severity classes according to the evaluations of magnetic resonance defaecography (MRD) in orthostatism.

\begin{tabular}{lccc}
\hline Pathology & Mild & Moderate & Severe \\
\hline Rectal prolapse (PCL) & 1 & 20 & 26 \\
Bladder prolapse (PCL) & 5 & 15 & 17 \\
Iato pubo-rectal hiatus enlargement & 3 & 13 & 28 \\
Pelvic floor descent & 3 & 18 & 26 \\
Rectal prolapse (H Line) & 18 & 22 & 7 \\
Bladder prolapse (H line) & 25 & 9 & 3 \\
Anterior rectocoele & 10 & 27 & 3 \\
Posterior rectocoele & 8 & 3 & 1 \\
\hline
\end{tabular}

PCL, pubo-coccygeal line. 


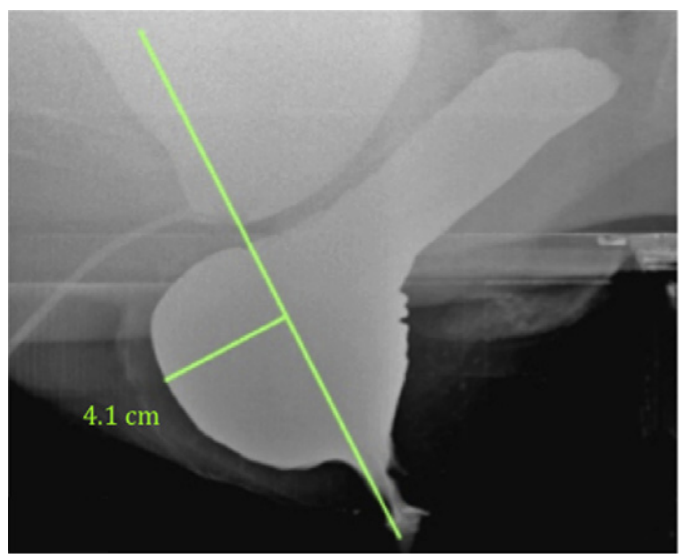

(a)

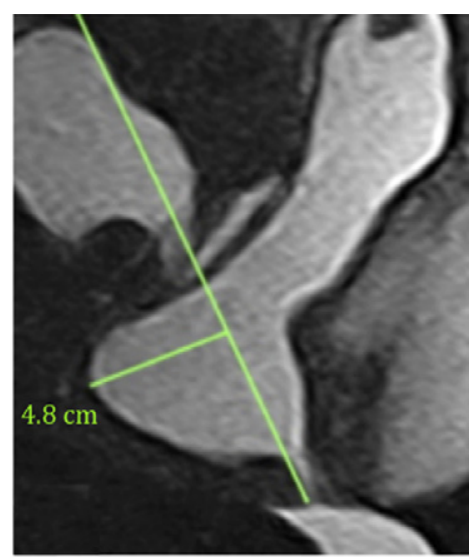

(b)

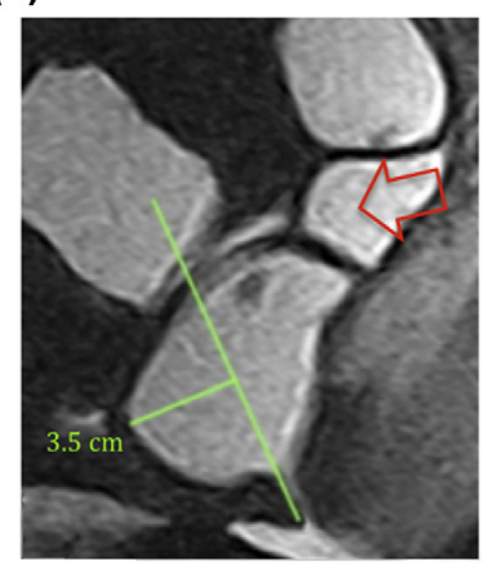

(c)

Figure 1 Comparison between CD (a) and MRD in orthostatism (b) and in the supine position (c) during evacuation. The measurement of the anterior rectocoele is overestimated by MRD in orthostatism and underestimated by MRD in the supine position, compared to the CD. In (c) a mucosal intussusception of the posterior wall of the rectum (arrow) is visible and was not detected by CD.

Excellent concordance values were obtained for measurements of the anterior rectocoeles and the ARAs with the ICC of 0.86 during straining and 0.87 during defaecation for the first, 0.80 during straining and 0.82 at rest, and during maximal contraction and evacuation for the latter, respectively (Fig 4).

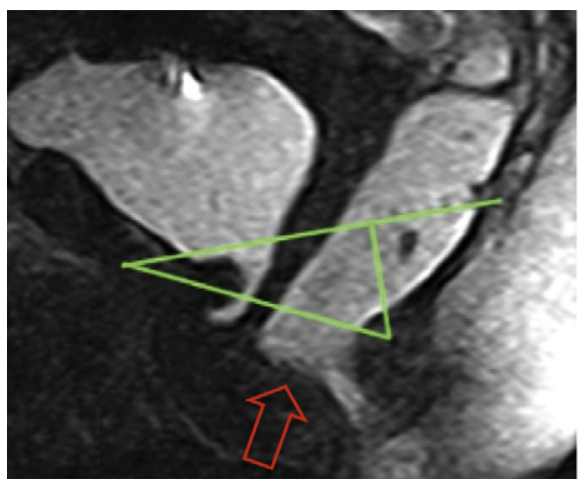

(a)

\section{Discussion}

Currently, the surgical planning of PFD is based on physical examination, functional tests, and data obtained with standard $C D$. More recently, in order to improve

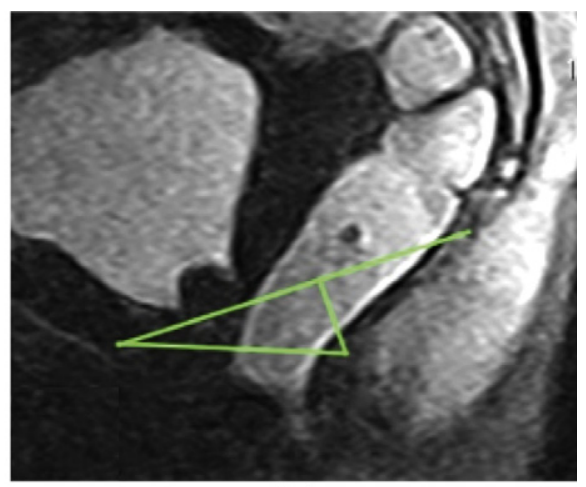

(b)

Figure 2 Comparison between MRD in orthostatism (a) and in the supine position (b) during evacuation. The evaluation of pelvic organ prolapse below the PCL and the $\mathrm{H}$ line shows an underestimation of MRD in the supine position either in the anterior and the posterior compartments. Also the anterior rectocoele is more evident in the tomograms acquired in orthostatism (arrow). 


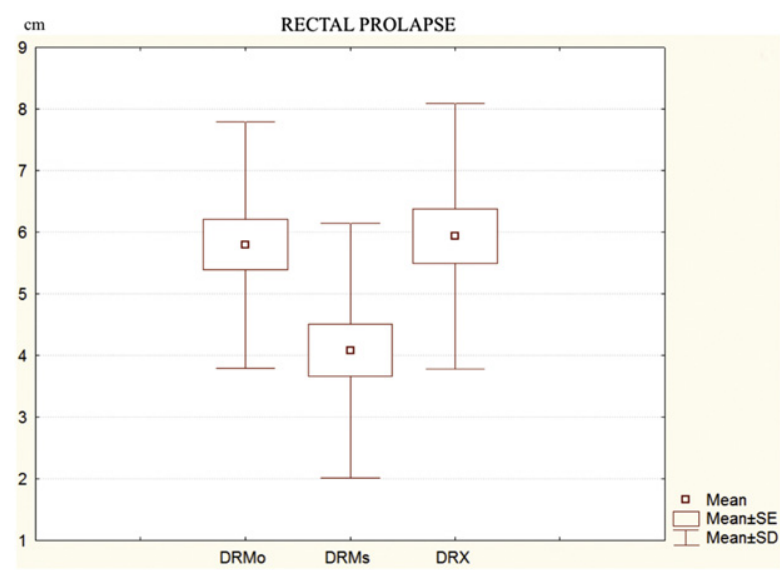

(a)

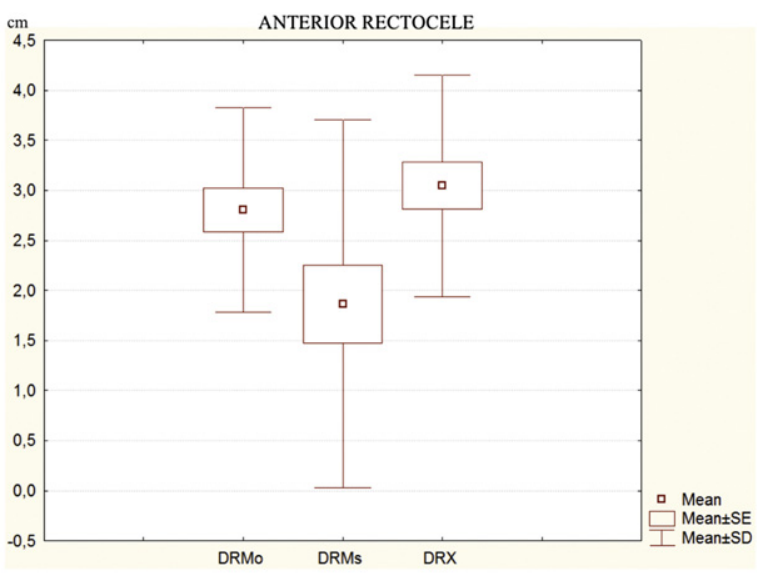

(b)

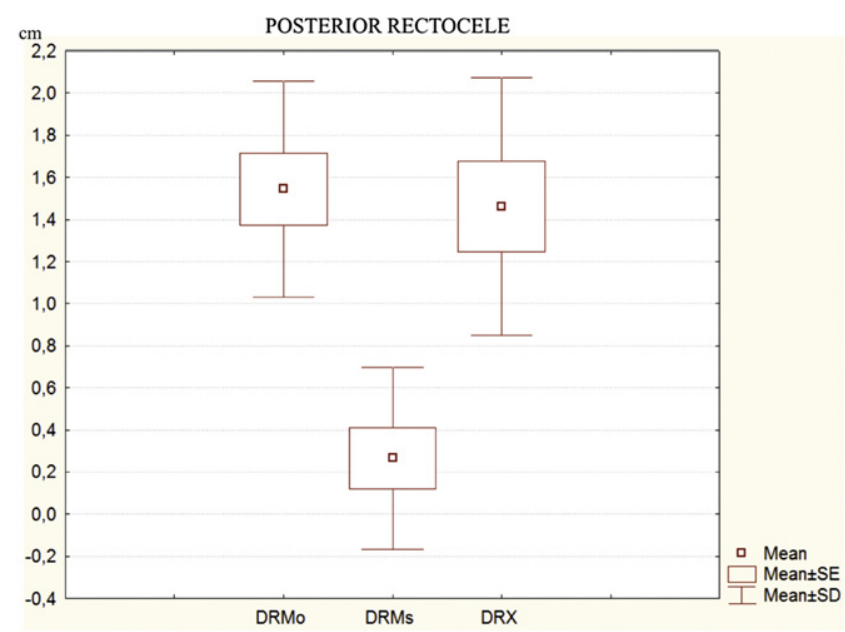

(c)

Figure 3 The values found for rectal prolapse (a), anterior rectocoele (b) and posterior rectocoele (c) in MRDs show an underestimation of the values compared both to MRDo and to CD.

diagnostic accuracy, the data obtained from conventional tests have been integrated with MRD imaging, which allows a complete pelvic floor assessment and a tailored surgical approach.

Most of the reports dealing with this subject refer to closed magnet systems with high field $(1.5 \mathrm{~T})$ and T2weighed acquisitions on multiple planes, with the patient

Table 7

Wilcoxon sign test.

\begin{tabular}{llllrc}
\hline Pathology & $\begin{array}{l}\text { No. of } \\
\text { patients }\end{array}$ & MRDo & MRDs & DRX & $p$-value \\
& 24 & $5.8 \pm 2$ & $4.1 \pm 2.1^{\mathrm{b}}$ & $5.9 \pm 2.2$ & $<0.0001$ \\
\hline Rectal prolapse & 24 & $2.8 \pm 1$ & $1.9 \pm 1.8^{\mathrm{c}}$ & $3 \pm 1.1$ & $<0.0001$ \\
Anterior rectocoele & 22 & $1.5 \pm 0.5$ & $0.3 \pm 0.4^{\mathrm{d}}$ & $1.5 \pm 0.6$ & 0.001 \\
\hline Posterior rectocoele & 9 & &
\end{tabular}

MRDo, magnetic resonance defaecography in orthostatism; MRDs, magnetic resonance defaecography in the supine position; $C D$, conventional defaecography.

a Analysis of variance.

b $p<0.0001$ versus MRDo and CD.

${ }^{c} p<0.001$ versus MRDo and CD.

d $p=0.008$ versus MRDo and $p=0.01$ versus CD. in the supine position or lying on a side with flexed knees. ${ }^{3,5}$ Broekhuis et al. ${ }^{11}$ used a closed magnet, with high field ( $\left.3 \mathrm{~T}\right)$, T2-weighed acquisitions on the sagittal plane and the patient in the supine position with flexed knees.

The introduction of $0.5 \mathrm{~T}$ open magnets, with dedicated devices and wide enough gantry, allows MRD with the patient sitting. ${ }^{3,12}$ However, existing reports compare MRD examinations performed in two different positions used MR systems with different static magnetic field intensity (1.5 T for supine and $0.5 \mathrm{~T}$ for the sitting position). This method strongly reduces the possibility of comparing the respective results because of the different magnet performances and sequences used. ${ }^{3,5}$

To our knowledge, the present study is the first to perform MRD in the orthostatic position. In the present study a newly designed permanent open magnet with static field of $0.25 \mathrm{~T}$, supplied with a table tilting from $0^{\circ}$ to $90^{\circ}$ was used, which facilitates the same examination protocol in the two different positions, using the same technical conditions, unlike other comparable studies.

The choice to provide an $80^{\circ}$ pitch to the table during the orthostatic examination was made in order to make 


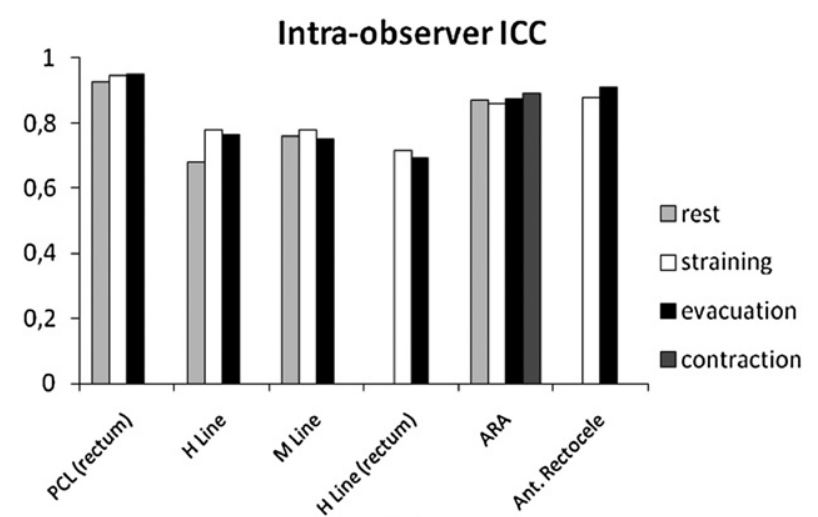

(a)

Inter-observerICC

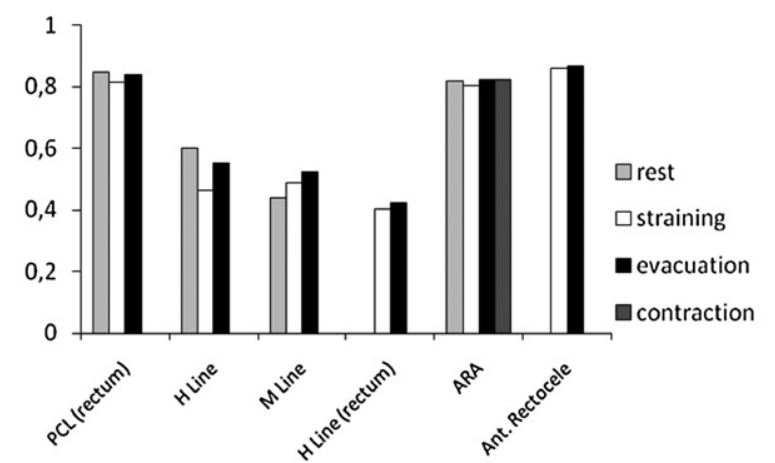

(b)

Figure 4 Visual representation of intra-observer (a) and interobserver (b) ICC values for all the measurements taken by the tomograms obtained with the MRD examination in orthostatism at rest, and during maximal contraction, straining and evacuation, respectively.

patients feel more stable and to reduce movement artefacts into the upright position. Despite this, some patients felt uneasy about the unusual position, and benefited from repeated straining manoeuvres and encouragement by the medical staff.

A further advantage results from the magnet being permanent, so that the costs of installation, management, and maintenance are lower than those of superconducting magnets.

As in $C D$, static sequences were acquired at rest, and during straining and contraction.

The TFE T1-weighed sequences, usually used for the dynamic study of the knee, were adjusted because the steady state free precession (balanced) sequences, normally employed for the evacuative study, were unavailable on the authors' system.

An acceptable balance was achieved between spatial resolution, temporal resolution, and signal intensity, and the optimized dynamic sequence had an acquisition time of $3 \mathrm{~s}$ /image. Another reduction of temporal resolution resulted from the need to a $2 \mathrm{~s}$ break between one image acquisition and the following acquisition to calibrate the image.

A better temporal resolution could probably be obtained either by dedicated sequences for the MRD free from a calibration break and with improved signal-to-noise ratio to reduce acquisition time or by the setting of dynamic sequences like steady state free precession (balanced).

The use of the 3D HYCE, GE balanced sequence, allowed a preliminary morphological study of the pelvic floor and pelvic organs in a static multiplanar acquisition. The morphological assessment appears to be extremely useful. In one case, for example, it confirmed the presence of herniation of the anterior rectal wall, previously suspected at $C D$, and to establish its precise para-median position, dimensions, and morphology. Additionally, images oriented on the axial plane enabled the evaluation of the puborectalis hiatus enlargement in case of pelvic floor weakness.

In the present population, the comparison between the orthostatic and the supine study showed statistically significant differences, for almost all the measurements obtained except for the ARA. Thus appears to be influenced only by the function of the pubo-rectalis muscle, regardless changes of position.

The present results show that MRD in the supine position is less accurate in the assessment of pelvic organ prolapse and pelvic floor weakness, underestimating the number and width of anterior rectocoeles, in line with the literature. ${ }^{7,11}$

A further limitation of the supine position is represented by the difficulty of carrying out the voiding phase, which is the most important phase for correct clinical-diagnostic assessment. In the present population, only 28 of the 49 patients enrolled managed to complete the MRD in the supine position, whereas all patients performed the voiding phase in the erect position.

With regards to the systems of evaluation, the present results showed an overestimation of measurements obtained using the PCL rather than the $\mathrm{H}$ line, regarding the severity of prolapses estimated by the respective scoring systems in both positions. In particular, 26 severe and 20 moderate prolapses were found using the first system compared to seven moderate and seven mild prolapses with the second. Similarly, an overestimation was observed in the evaluation of the anterior compartment with nine milder bladder prolapses diagnosed with the first system (Table 6).

These results could mean that the extent of prolapses below the PCL does not take into account the descent of the pelvic floor, whereas the $\mathrm{H}$ line represents the descent of the pubo-rectalis hiatus and gives a more accurate measurement of pelvic organ prolapse below the muscular plane. The HMO system is the more accurate method in the assessment of PFD because it considers the two different pathological aspects separately and it enables more correct clinical assessment and, consequently, gives more useful information for choosing the best treatment.

The comparison between $\mathrm{CD}$ and MRD was possible in all patients and was based on the evaluation of rectal prolapse below the PCL, anterior rectocoele and ARA, because it is impossible to measure the $\mathrm{H}$ line and the $\mathrm{M}$ line on the $\mathrm{CD}$ images. Conventional defaecograms are, in fact, a "cast" of the rectal lumen, and it is impossible to identify the posterior border of the pubo-coccygeal muscle, the position 
of which can only be estimated from the impression of contrast media on the posterior wall of the rectum.

In the present population, the comparison between $C D$ and MRD in the erect position gives similar results for almost every parameter measured. Even if a statistically significant difference was observed in the anterior rectocoeles measured in the voiding phase, a further evaluation of data following severity classes showed similar results between the two imaging techniques in all the patients examined. From these results it can be concluded that the force of gravity acts similarly in the semi-erect and in the sitting position, differently from supine position.

Unlike Lienemann et al., ${ }^{13}$ and Kelvin et al. ${ }^{14}$ who compared CD and MRD in the supine position and obtained similar results, the present data show statistically significant differences in the defaecatory phase both in the detection of prolapse of ARJ below the PCL, and in the measurement of anterior rectocoele width with an average underestimation by MRD.

The present data demonstrated that MR in the orthostatic position, although non-physiological, enables better assessment of PFD compared with MR performed in the supine position and with $C D$.

According to the literature, MRD has a key role, with more accurate results than $\mathrm{CD}$ in the preoperative assessment of the patient with pelvic floor weakness. ${ }^{2,15}$ Moreover, the advantage of MRD over CD is the assessment of soft tissues, and therefore, MRD enables an additional study of the pelvic organs and of the pelvic muscular floor. MRD also does not expose a person to ionizing radiation, an important aspect especially in women of child-bearing age (in the present population four patients were between 22 and 35 years of age). ${ }^{16}$

The repeatability of the measurements using MRD in different positions has been proved by calculating the intraobserver and interobserver concordance.

Only three papers regarding this subject have been published previously; compared to the present results, Morren et al. $^{17}$ reported lower intra-observer concordance values, whereas Fauconnier et al. ${ }^{18}$ demonstrated excellent both intra-and interobserver concordance. As for Broekhuis et al., ${ }^{11}$ in the present study an intra-observer concordance ranging from good to excellent for all measurements was observed. ${ }^{11}$

Although the number of intussusceptions detected in the present population was not high enough to make a statement about its statistical significance, the observations obtained suggest an advantage of CD compared with MRD. In particular, a mucosal intussusception, detected by $C D$, was not appreciated by MRD (Fig 1). This underestimation is likely attributable to the lower spatial and temporal resolution of sequences used for the dynamic study compared to serial imaging studies.

Ultimately in the present study work, abnormalities of the middle compartment were not observed, either with CD or with MRD, which was also related to the low population numbers.

In conclusion, MRD is feasible and of high diagnostic quality if performed on a $0.25 \mathrm{~T}$ open magnet. The erect study, even if not physiological, is more accurate in the evaluation of PFD compared with assessment in the supine position, which is sometimes hampered by the difficulty to complete the evacuation phase.

The scoring system HMO in the study of PFD provides the most reliable method because it separately evaluates two different conditions, which often coexist (pelvic floor weakness and pelvic organ prolapse), and therefore, it enables a more complete clinical assessment, giving essential information for the best choice of treatment.

MRD compared to $C D$, allows assessment of soft tissues thus offering a simultaneous assessment of pelvic organs and muscular structures of pelvic floor, through multiplanar acquisitions.

The absence of risks related to exposure to radiation makes MRD a candidate for the initial study for younger patients.

\section{References}

1. Olsen AL, Smith VJ, Bergstrom JO, et al. Epidemiology of surgically managed pelvic organ prolapse and urinary incontinence. Obstet Gynecol 1997;89:501-6.

2. Boyadzhyan L, Raman SS, Raz S. Role of static and dynamic MR imaging in surgical pelvic floor disfunction. RadioGraphics 2008;28:949-67.

3. Mortele KJ, Fairhurst J. Dynamic MR defaecography of the posterior compartment: indications, techniques and MRI features. Eur J Radiol 2007;61:462-72.

4. Pannu HK, Kaufman HS, Cundiff JW, et al. Dynamic MR imaging of pelvic organ prolapse: spectrum of abnormalities. RadioGraphics 2000;20:1567-82.

5. Fielding JR. Practical MR imaging of female pelvic floor weakness. RadioGraphics 2002;22:295-304.

6. Fiaschetti V, Squillaci E, Pastorelli D, et al. Dynamic MR defaecography with an open-configuration, low-field, tilting MR system in patients with pelvic floor disorders. Radiol Med 2011;116:620-33.

7. Bertschinger KM, Hetzer FH, Roos JE, et al. Dynamic MR imaging of pelvic floor performed with patient sitting in an open-magnet unit versus with patient supine in a closed-magnet unit. Radiology 2002;223:501-8.

8. Barbaric ZL, Marumoto AK, Raz S. Magnetic resonance imaging of the perineum and pelvic floor. Top Magn Reson Imaging 2001;12:83-92.

9. Kruyt RH, Delemarre JB, Doornbos J, et al. Normal anorectum: dynamic MR imaging anatomy. Radiology 1991;179:159-63.

10. Bump RC, Mattiason A, Bø K, et al. The standardization of terminology of female pelvic organ prolapse and pelvic floor dysfunction. Am J Obstet Gynecol 1996;175:10-7.

11. Broekhuis SR, Kluivers KB, Hendriks JC, et al. Dynamic magnetic resonance imaging: reliability of anatomical landmarks and reference lines used to assess pelvic organ prolapse. Int Urogynecol J Pelvic Floor Dysfunct 2009;20:141-8.

12. Schoenenberger AW, Debatin JF, Guldenschuh I, et al. Dynamic MR defaecography with a superconducting open-configuration MR system. Radiology 1998;206:641-6.

13. Lienemann A, Anthuber C, Baron A, et al. Dynamic MR colpocystorectography assessing pelvic floor descent. Eur Radiol 1997;7:1309-17.

14. Kelvin FM, Maglinte DD, Hale DS, et al. Female pelvic organ prolapse: a comparison of triphasic dynamic MR imaging and triphasic fluoroscopic cystocolpoproctography. AJR Am J Roentgenol 2000;174:81-8.

15. Goodrich MA, Webb MJ, King BF, et al. Magnetic resonance imaging of pelvic floor relaxation: dynamic analysis and evaluation of patients before and after surgical repair. Obstet Gynecol 1993;82:883-91.

16. Goei R, Kemerink G. Radiation dose in defecography. Radiology 1990;176:137-9.

17. Morren GL, Balasingam AG, Wells JE, et al. Triphasic MRI of pelvic organ descent: sources of measurement error. Eur J Radiol 2005;54:276-83.

18. Fauconnier A, Zareski E, Abichedid J, et al. Dynamic magnetic resonance imaging for grading pelvic organ prolapse according to the International Continence Society classification: which line should be used? Neurourol Urodyn 2008;27:191-7. 